\title{
Coupling of microwave to convective drying for improving fruit quality
}

\author{
Bahloul, N. ${ }^{a^{*}}$; Balti, M. A. ${ }^{\text {; }}$ Guellouze, M. S. ${ }^{b}$; Kechaou, N. ${ }^{a}$ \\ ${ }^{a}$ Groupe de Recherche en Génie des Procédés Agro-alimentaires, Laboratoire de Mécanique des \\ Fluides Appliquée, Génie des Procédés et Environnement, Ecole Nationale d’Ingénieurs de Sfax, \\ Université de Sfax, BP 1173, 3038, Sfax, Tunisia. \\ b Laboratoire d'Etudes des Systèmes Thermiques et Energétique, Ecole Nationale d’Ingénieurs de \\ Monastir, Université de Monastir, Tunisia.
}

*E-mail of the corresponding author: neilabahloul@yahoo.com

\begin{abstract}
The present study aims to find the best drying method to minimize the duration of the operation while respecting the nutritional value of the product. Experiments of convective drying and microwave radiation were respectively carried out with a convective dryer (heat temperature: $65{ }^{\circ} \mathrm{C}$ and air velocity: $1.3 \mathrm{~m} / \mathrm{s}$ ) and a microwave oven (power level: $100 \mathrm{~W}$ and frequency: $2450 \mathrm{MHz}$ ). Six coupling tests between the convective drying and microwave radiation were conducted. The only variable condition is the time output of tomatoes from the convective dryer or the microwave oven corresponding to an intermediate mass ratio (IMR) 0.5, 0.4 and 0.3. The microwave drying removes the bound water faster than convective drying. This explains the observed time savings due to convection-microwave and microwave-convection combined drying. The combination of convection drying and microwave drying to an IMR 0.5 preserved better the red color and the antioxidants of the tomato. Thus, the convection-microwave combined drying (IMR 0.5) proved most suitable for preserving the quality of tomatoes.
\end{abstract}

Keywords: convective drying; microwave; coupled drying; color. 


\section{Introduction}

Tunisia is ranked among the top ten countries in the world in terms of tomato processing, with a local production of 1.2 million tons of tomatoes in 2013, fifty thousand tons of which are destined for processing [1]. Furthermore, the export of dried tomatoes has shown remarkable growth in recent years. Tomato is an herbaceous climacteric fruit, which belongs to the order of Solanale, the family of Solanaceae and the genus of Lycopersicon. It is native to the northwestern South of America and it is grown in warm and temperate countries around the world. According to some studies, regular consumption of tomatoes or tomato products can reduce the risk of cancer, as well as cardiovascular diseases, diabetes and osteoporosis [2],[3]. Indeed, Tomato is a fruit which is rich in antioxidant compounds, and more particularly, in polyphenols and vitamin C. The transformation processes involve one or more heat treatments which can affect the levels of antioxidants and thus the nutritional quality of the products. In fact, these micro-constituents can be partially degraded under the effect of heat and light. In this context, our study aims to find the best mode for drying tomatoes by combining convective drying and microwaves in order to minimize the duration of the operation while respecting the nutritional value of tomatoes.

\section{Materials and Methods}

\subsection{Raw material}

The tomato samples used for drying are "round" varieties grown under a greenhouse and supplied from the "AGRIFOOD" Tunisian company.

\subsection{Determination of drying kinetics}

\subsubsection{Convective drying experiments}

The drying kinetics of the tomatoes were performed using the convective drying loop of the research group GP2A (ENIS). After setting the air flow, the controller starts to heat up to the set temperature. Once the experimental conditions are stable, the samples to be dried are washed and cut into quarters. Then, the samples are placed on a suitable support; this support is suspended in the horizontal test vein by a hook located below a precision balance. The drying kinetics are determined through the measurements of the mass variation of the samples over time by means of a digital precision electronic scale. This balance is linked to a computer and equipped with a data output enabling the acquisition of the mass over time by means of a suitable software. The experimental conditions carried out with the convective drying loop are fixed at a temperature of $65^{\circ} \mathrm{C}$., an air velocity of $1.3 \mathrm{~m} / \mathrm{s}$ and a final mass ratio of 0.2 , imposed by the manufacturer.

\subsubsection{Microwave drying experiments}

The drying experiments were carried out in a domestic microwave oven at a frequency of $2450 \mathrm{MHz}$ and three power levels (100, 300 and $600 \mathrm{~W}$ ) for a cycle of 15 seconds. The 
drying end criterion is also the final mass ratio of 0.2 . Preliminary tests at different power levels were carried out and led to the choice of the lowest power $(100 \mathrm{~W})$ for subsequent experiments based on the visual appearance of tomatoes during drying.

\subsubsection{Experimental protocol of convective and microwave coupled drying}

Six coupling tests were carried out under the same drying conditions as: a temperature of 65 ${ }^{\circ} \mathrm{C}$., an air velocity of $1.3 \mathrm{~m} / \mathrm{s}$, a power of $100 \mathrm{~W}$ and a final mass ratio of 0 , 2. The only variable condition is the intermediate output time from the convective dryer or the microwave oven corresponding to an intermediate mass ratio (IMR) of 0.5; 0.4 and 0.3. This ratio corresponds to the breaking point and the change from one drying mode to another:

- 3 assays of coupling convective drying (Co) to microwave radiation (Mo) at 3 IMR: 0.5; 0.4 and 0.3 .

- 3 other coupling tests by switching from microwave drying (Mo) to convective drying (Co) at 3 IMR: 0.5; 0.4 and 0.3.

\subsection{Physicochemical analysis}

\subsubsection{Preparation of extracts}

The extraction is carried out using an ultrasonic bath. One gram of crushed tomatoes is mixed with $50 \mathrm{ml}$ of pure ethanol for 2 hours. The solvent was evaporated on a rotary evaporator. Dry extracts stored at $4^{\circ} \mathrm{C}$ are obtained until use.

\subsubsection{Water content}

The water content, $\mathrm{X}$ (kg of water / kg M.S.) is calculated using the following equation:

$$
\mathrm{X}=\frac{\mathrm{m}-\mathrm{ms}}{\mathrm{ms}}
$$

with: m: mass of the wet sample (kg), ms: mass of the dry sample $(\mathrm{kg})$. 2.3.3. Ascorbic acid analysis

The ascorbic acid content is measured by the 2,6-dichloroindophenol titration method [4].

\subsubsection{Color analysis}

The color is measured using a colorimeter (Konica Minolta CR 300) with CIE Lab system (L *, a*, b*). Statistical analysis was performed using SPSS software (version 13, Inc. SPSS, Chicago, USA) at a confidence interval of $95 \%$. 


\section{Results and discussion}

\subsection{Kinetics of convective and microwave drying}

Figure 1 shows the evolution of the reduced water content as a function of the drying time for the convective $\left(65^{\circ} \mathrm{C}\right.$. and $1.3 \mathrm{~m} / \mathrm{s}$ ) (FIG. 1 a) and for microwaves drying (at $100 \mathrm{~W}$ ) (FIG. 1b). The drying time needed to obtain a final mass ratio of 0.2 (i.e. a final water content of $0.25 \mathrm{~kg}$ water $/ \mathrm{kg} \mathrm{DM}$ ) is 5 hours and 21 minutes. The water content decreases rapidly at the first time and then more and more slowly at the end of the drying. Indeed, at the beginning of drying, the mechanism of water displacement through the pores walls of the solid towards the surface is carried out in an accelerated manner. At the end of the drying process, increasing the temperature in the center of the product and decreasing its water content result in a new state of equilibrium in the product. Thus, water is increasingly hard to migrate into the product and the internal transfer of matter becomes a limiting phenomenon [5].
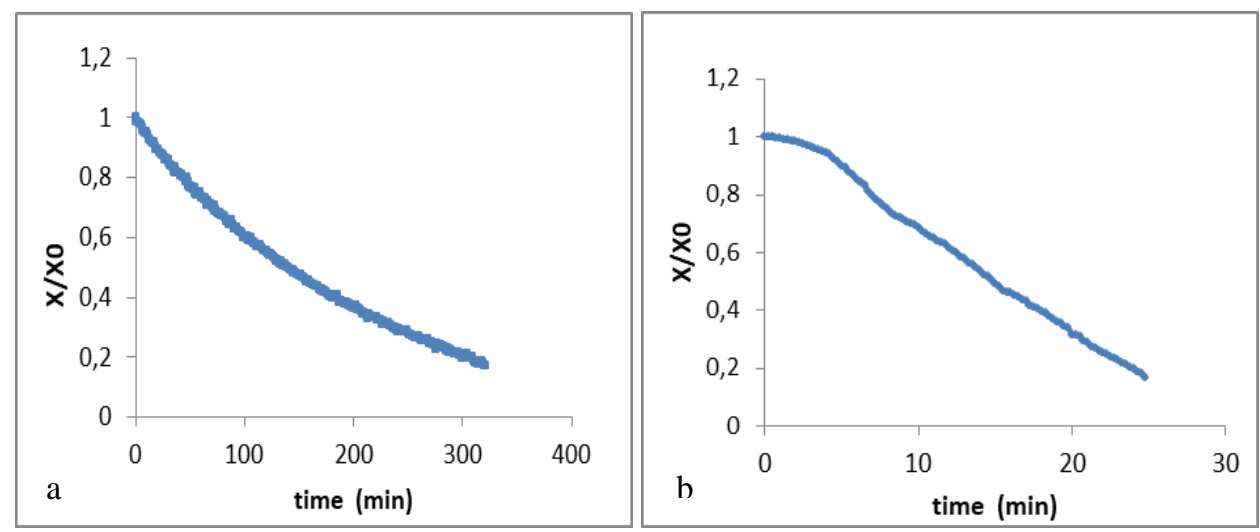

Figure 1: Evolution of the reduced water content as a function of the drying time by: (a) forced convection drying at $65^{\circ} \mathrm{C}$ and $1.3 \mathrm{~m} / \mathrm{s}(\mathrm{XO}=24.263 \mathrm{~kg}$ water $/ \mathrm{kg} \mathrm{M.S}) ;$ and (b) microwaves radiation at $100 \mathrm{~W}(\mathrm{XO}=24.258 \mathrm{~kg}$ water $/ \mathrm{kg} \mathrm{M} . S)$

Microwave drying at a relatively low power $(100 \mathrm{~W})$ allows to achieve the same mass ratio of 0.2 (i.e. a final water content of $0.25 \mathrm{~kg}$ of water / kg DM) in 25 minutes; that means a gain of 4 hours and 56 minutes compared to convective drying. Despite the short duration of microwave treatment and the visual aspect, the sample had a softer texture and showed intense yellowing of the skin that we did not notice for convection dried samples; hence, the idea of combining convective drying with microwaves seems to be interesting. 


\subsection{Coupled drying}

Figure 2 shows the evolution of the reduced water content as a function of time for the various alternating convection / microwave and microwave / convection drying modes. In the early stages of drying, the kinetic rates obtained by convection-microwave coupled drying at different IMR are similar to that of convective drying. After that, the water content decreases rapidly under the effect of microwave radiation compared to the convective drying applied alone.

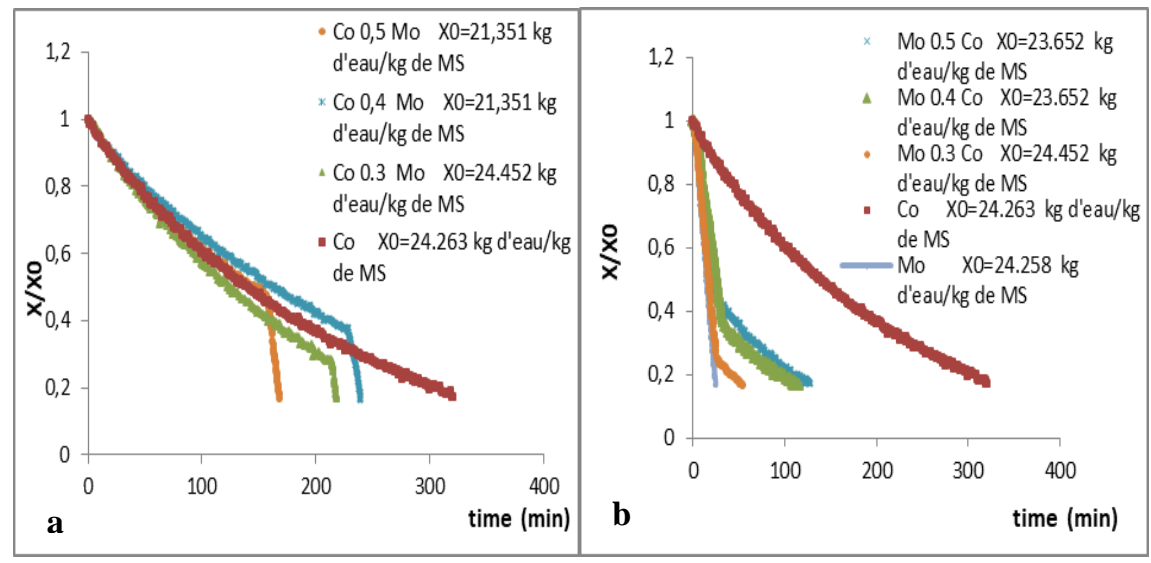

Figure 2: Evolution of the reduced water content as a function of the drying time obtained by (a) convection-microwave coupled drying, (b) microwave-convection coupled drying.

Thus, microwave drying allows removing the weak bonded water more rapidly than convection drying. In the beginning, the kinetic rates obtained by microwave-convection coupled drying at different IMR are similar to that of microwave drying; while at the end of drying, they are comparable to that of convective drying. Microwave radiations permit to reduce the water content of the product more quickly than convective drying. This fact results in a remarkable reduction of the drying time compared to that of convection drying and convection-microwave coupled drying.

\subsubsection{Drying for an intermediate mass ratio of 0.5}

The evolution of the reduced water content as a function of drying time is shown in Figure 3 for different convection / microwave and microwave / convection coupled drying and at each IMR. It is noted that microwave radiations followed by convective drying (Mo $0.5 \mathrm{Co}$ ) reduce the drying time by 3 hours and 13 minutes compared to convective drying (Co). While convective drying followed by microwave radiation (Co $0.5 \mathrm{Mo}$ ) allows decreasing the duration by 2 hours and 33 minutes. 

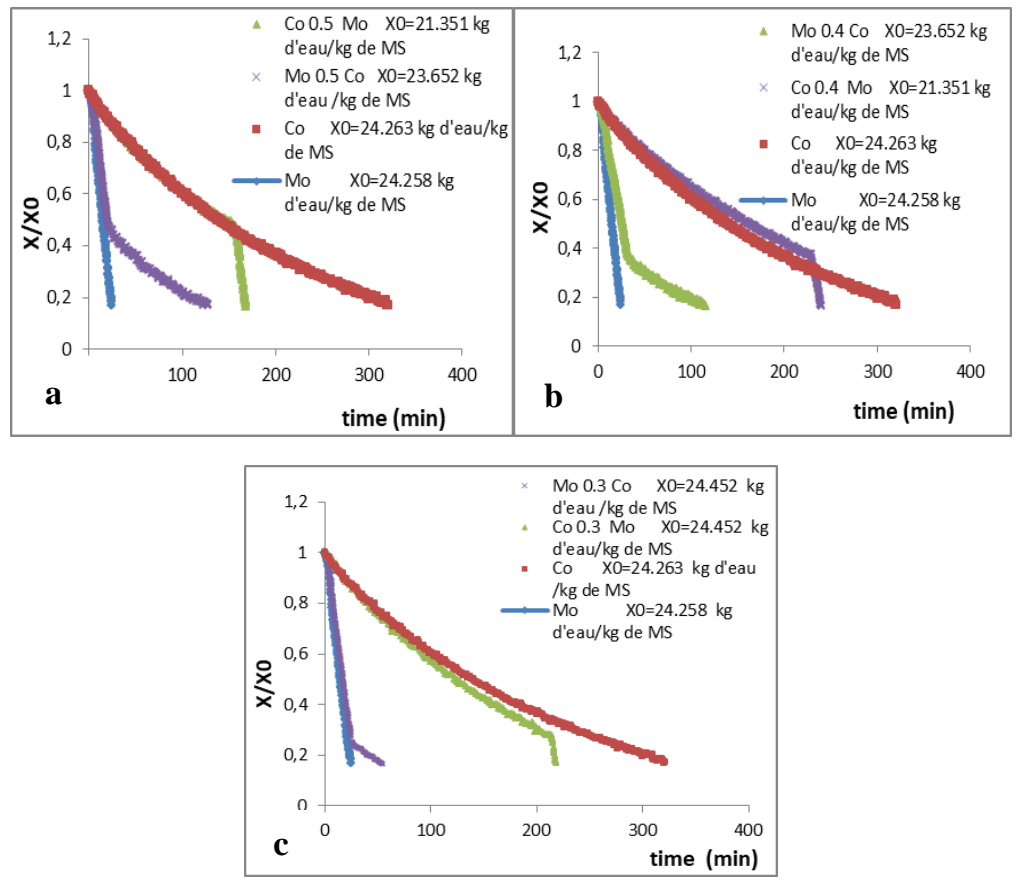

Figure 3: Evolution of the reduced water content as a function of time for the convection / microwave and microwave / convection dryings at: (a) IMR of 0.5; (b) IMR of 0.4 and

(c) IMR of 0.3

\subsubsection{Drying for an intermediate mass ratio of 0.4}

The coupled drying which starts with microwave radiation (Mo 0.4 Co) lasts 1 hour and 55 minutes, ie a gain of 2 hours and 4 minutes compared to the convective-microwave drying (Co $0.4 \mathrm{Mo}$ ) and 3 hours and 25 minutes compared to convective drying applied alone. The convection-microwave coupled drying lasts 3 hours and 59 minutes, thus reducing the drying time of 1 hour and 21 minutes compared to the convective drying.

\subsubsection{Drying for an intermediate mass ratio of 0.3}

The drying duration obtained by microwave-convection drying (Mo $0.3 \mathrm{Co}$ ) is $54 \mathrm{~min}$, giving a gain of $4 \mathrm{~h} 26 \mathrm{~min}$ with respect to the convective drying. The convectionmicrowave drying (Co $0.3 \mathrm{Mo}$ ) allows a gain of $1 \mathrm{~h} 42$ min compared to the convective drying (drying time is equal to $3 \mathrm{~h} 38 \mathrm{~min}$ ). Based on the drying time criterion, which is the most important for the manufacturer, we can opt for microwave-convection drying at this stage. However, the visual appearance of dried tomatoes by convection- microwave mode seems to be better. Therfore, biochemical analyzes were carried out on dried tomatoes. 


\subsection{Determination of quality parameters}

\subsubsection{Color analysis}

Measurement of color by referring to the CIE Lab coordinates ( $\mathrm{L}^{*}$, $\mathrm{a} *$ and $\mathrm{b} *$ ) shows that luminance $\left(\mathrm{L}^{*}\right.$ ) of dried tomatoes is lower than that of fresh tomato (Figure 4). This fact can be explained by the non-enzymatic browning during drying. The values corresponding to the convection-microwave drying with a transition mass ratio of 0.5 and 0.4 are close to that of fresh tomato. Regarding the $\left(\mathrm{a}^{*}\right)$ index, it is noted that the values obtained after convective-radiative drying of tomatoes at transition ratios of 0.5 and 0.4 are significantly greater than those obtained for fresh tomato. The $\left(b^{*}\right)$ values obtained after microwave, microwave-convective drying and convective drying of tomatoes increase significantly with respect to that of fresh tomato.

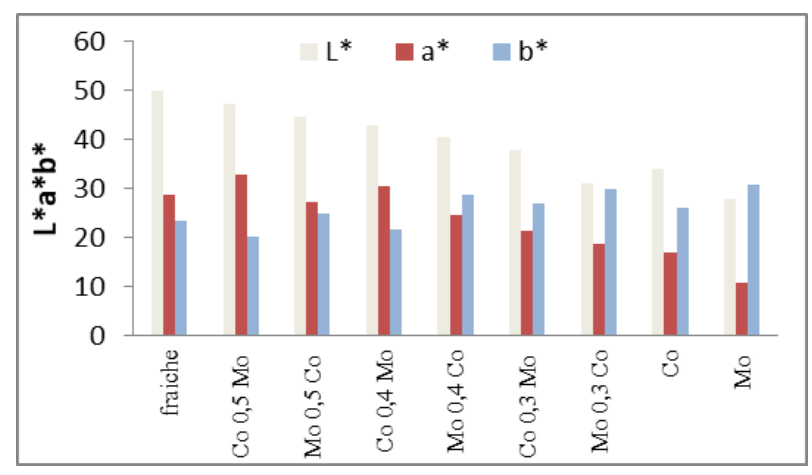

Figure 4: Effect of drying on the color of tomatoes

\subsubsection{Ascorbic acid}

The ascorbic acid contents of the dehydrated tomatoes are summarized in Table 1 . As a result of the heat treatments undergone by the tomato, the vitamin $C$ content is reduced in comparison with that of fresh product. The convective-microwave dryings, for IMR of 0.5 ; 0.4 and 0.3 , lead to vitamin $\mathrm{C}$ loss due to tomato exposure to heated air $\left(65^{\circ} \mathrm{C}\right)$. After microwave-convection drying, vitamin $\mathrm{C}$ loss was greater in dried tomatoes than in other samples. Thus, convective-microwave (Co $0.5 \mathrm{Mb}$ ) drying better preserves ascorbic acid in tomatoes.

\section{Conclusions}

Microwave drying eliminates weak bonded water faster than convection drying. This explains the saving in time observed after convection-microwave and microwaveconvection drying. Based on the nutritional quality attributes of the tomato, the convection- 
microwave combined drying (IMR 0.5) proved most suitable for preserving the quality of tomatoes.

Table 1: Ascorbic acid contents of tomatoes (mg / $100 \mathrm{~g} \mathrm{DM)}$

\begin{tabular}{lc}
\hline Drying conditions & Ascorbic acid content \\
\hline Fresh & $384.441 \pm 0.175$ \\
Co 0.5 Mo & $234.375 \pm 0.022$ \\
Mo 0.5 Co & $111.877 \pm 0.023$ \\
Co 0.4 Mo & $136.491 \pm 0.021$ \\
Mo 0.4 Co & $102.387 \pm 0.011$ \\
Co 0.3 Mo & $130.293 \pm 0,011$ \\
Mo 0.3 Co & $97.286 \pm 0.000$ \\
Co & $109.905 \pm 0.011$ \\
Mo & $81.394 \pm 0.023$ \\
\hline
\end{tabular}

\section{Acknowledgments}

This study was financed by the National Agency for Energy Control in the context of a Federated Research Project.

\section{References}

[1] FAO, 2013, Faostat.fao.org

[2] Davies, J.N. ; Hobson, G.E. The constituents of tomato fruit-the influence of environment. nutrition. and genotype. CRC Critical Reviews in Food Science and Nutrition 1981, 15, 205-280.

[3] Rao, A.V. Lycopene Content of Tomato Products: Its Stability, Bioavailability and In Vivo Antioxidant Properties. Food and nutrition research 2006, 10, 1016-1043.

[4] AOAC, Officials Methods of Analysis. Vitamin C (ascorbic acid) in vitamin preparations and juices: 2, 6 dichloroindophénol titrimetric method final action. 43.064 and 43.065, 1984

[5] Bahloul, N.; Boudhrioua, N.; Kouhila, M.; Kechaou N. Drying characteristics of convective solar drying of olive leaves. Journal of Food Process Engineering 2009, 34 (4), 1338-1362. 